\title{
Simulations of Gas Dispersion Arising From Gas Venting Process Using Cellular Automata
}

\author{
Agus. Yodi Gunawan, Rio Harapan, Aditya. Firman Ihsan, Darmadi Soetikno
}

\begin{abstract}
For maintenance purposes, gas inside the pipeline need to be emptied by venting process. The gases that emitted during the venting process may cause negative impact to the environment and may travel to various receptors depending on the meteorological conditions. Study of dispersion model from venting process is intended to predict the maximum concentration in ambient air and to predict safe area radius. In this study, a gas dispersion model is constructed using cellular automata (CA) with some specific related rules. Our rules cover parameters that play important roles for gas dispersion such as wind current, land topography, and rate of dispersion. The present results may help practitioner to predict the safe area radius to avoid, for instance, the explosion surrounding venting process.
\end{abstract}

Keywords-Cellular automata, ambient air, maximum concentration, safe area radius, venting process.

\section{INTRODUCTION}

$\mathrm{F}$ OR maintenance purpose, every 2 year the segment of transmission pipeline need to be replace with the new one. Due to safety reason, the segmentation of pipeline needs to be depressurizing and emptying first before the replacement process been held. The depressurizing and emptying process was done by conducting venting process on the segment of pipeline.

The gas that emitted during venting process may cause negative impact to the environment and may travel to various receptors depending on the meteorological conditions. Once gasses emitted to ambient air, it is beyond our control and it would not be possible to control. The hydrocarbon gas that emitted during the venting process is a flammable gas. The dispersion of the hydrocarbon gases would increase the explosion risk of ambient air within the venting process been held. To better understand the dispersion of the gasses emitted from the vent to the ambient air, therefore study on dispersion modeling needs to be conducted.

The result of this study would be able to predict the maximum concentration in the ambient air and to predict the area that would be affected during the venting process. This result of this study also would be able to predict the safe

Agus. Yodi Gunawan is with the Mathematic Department, Institut Teknologi Bandung, Boulder, 40136 INA (corresponding author's phone: +62 222508126 ; fax: +62 22250 8126; e-mail: aygunawan@math.itb.ac.id).

Rio. Harapan is with Departement of Physics, Institut Teknologi Bandung, Bandung, 40135 INA, (e-mail: rioharapan@gmail.com).

Aditya Firman, Ihsan is with RC-Oppinet PPMS, Institut Teknologi Bandung, 40135 INA. (e-mail: aditya.fphoenix@gmail.com). radius during the venting process for specific venting location and to predict the seasonal impact on gas dispersion for specific venting location.

In section 2, we give a brief explanation on research methodology, and case study that been used for this study is given in section 3. Section 4 shows the results of the dispersion modeling. The conclusion of this research is given in Section 5. Nomenclature and references is given in the end of the paper.

\section{METHODOLOGY}

\section{A. Cellular Automata}

Cellular Automata (CA) is a mathematical model for computer simulation trying to model dynamical behavior. The concept of CA was first introduced by Von Neumann for a particular task, namely, to prove the existence of a universal computer capable of self-reproduction within a defined space. This concept has evolved into a theory leading to different types of CA, which have been applied to a variety of physical phenomena, as well as biological, chemical, etc.

From the systems framework, $\mathrm{CA}$ is a discrete dynamic system, i.e., space, time, and the system states are discrete. Each point in a regular space grid called cell may have any number of finite states. The cell states in the grid are updated based on a local rule, i.e., the state of a cell in a given time only depends upon its own previous state. All the cells within the grid are simultaneously updated, i.e., the whole grid state changes in discrete time steps.

\section{B. Rules Formulation}

To formulate the dynamics of gas dispersion using CA, we construct a square $n \times n \times m$ grid of cells, which each individual cell state represent the concentration of gas. Every cell is identified by its relative position using a simple $x-y-z$ coordinate. Suppose we denote a state of a cell in $(i, j, k)$ position with $A_{i j k}$. The finite automata of each cell is represented by an updated rule. With this rule, each cell state is computed, according to the state values of its neighbor cells.

Every cell state in this simulation is non-dimensional, so are all computations in the update rule.

Every cell has 6 neighbors, 4 adjacent horizontal neighbors (north, west, east, south) and 2 vertical neighbors (upward, downward). The update rule is formulated as follows 


$$
\begin{aligned}
& D\left(A_{i j k}^{t}\right)=c_{e}\left(A_{i, j+1, k}^{t}-A_{i j k}^{t}\right)+c_{e}\left(A_{i, j+1, k}^{t}-A_{i j k}^{t}\right)+ \\
& c_{e}\left(A_{i, j+1, k}^{t}-A_{i j k}^{t}\right)+c_{e}\left(A_{i, j+1, k}^{t}-A_{i j k}^{t}\right)+c_{u}\left(A_{i, j, k+1}^{t}-\right. \\
& \left.A_{i j k}^{t}\right)+c_{d}\left(A_{i, j, k-1}^{t}-A_{i j k}^{t}\right)
\end{aligned}
$$

where $c_{e}, c_{w}, c_{n}, c_{s}, c_{u}$ and $c_{d}$ are the dispersion coefficients of each direction respectively. We then can determine the cell state at time $t+1$ as

$$
A_{i, j, k}^{t+1}=A_{i j k}^{t}+D\left(A_{i j k}^{t}\right)
$$

The value of the dispersion coefficients will be determined by following factors.

\section{Horizontal Diffusion}

Horizontal diffusion produces mass interchanges between neighbor cells due only to concentration gradients. It is assumed that there exists an isotropic diffusion, i.e., the mass diffusion is the same in all 4 horizontal directions.

\section{Wind Field}

Mass of the gasses can be transported by any occurring wind in the area. It will affect the dispersion towards horizontal direction.

\section{Turbulent Velocity}

The impact of turbulence upon the concentration of the venting gas in a given cell $(i, j, k)$ must also be considered; even more so in the case of dense gases. This will affect the upward dispersion coefficient

\section{Land topography}

We assume that heavy gas tends to disperse faster if there is no obstacle in horizontal direction, especially towards a valley.

\section{Gas outburst}

The gas is released through a small venting hole from a very high pressure vessel. We then can expect that there will be significant outflow velocity in the source point. This will also affect the upward coefficient together with turbulent velocity.

\section{SIMULATION}

\section{A. Gas Venting Process}

We assume that gas venting process results a continuous release of gas with constant concentration until the pressure inside pipeline reaches atmospheric pressure. The location of the venting line is on cell $(10,10)$. Furthermore, we set in our simulation that the gas is released through a small venting pipe on ground level until $t=50$ with released concentration is valued by 1 for every $t$. Although CA model we formulate is 3-dimensional, by means of practicality, we only show the result in 2-dimensional slice of a specific altitude. In this study, we will observe $20 \times 20$ grid area on ground level around venting pipe

\section{B. Case Study}

In this study, we tried 2 cases for gas dispersion simulation. The first case will assume plain land topography with a generated wind field, while the second case will assume the absence of wind to any direction with simple generated land topography. The generated wind field and land topography for each case are shown in Figure 1 and Figure 2 respectively.

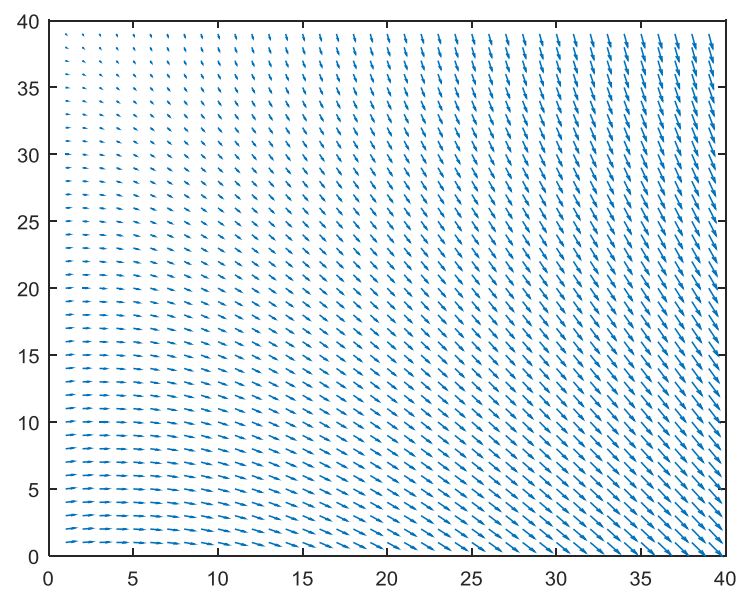

Fig. 1 Generated wind field for case 1 simulation

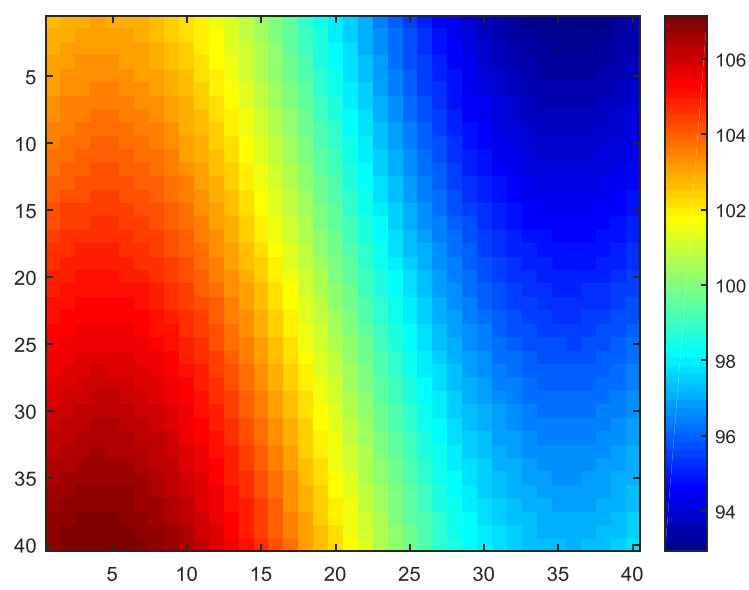

Fig. 2 Generated land topography for case 2 simulation

\section{RESULTS AND DISCUSSION}

By running the $\mathrm{CA}$ model with the rule formulated by all affecting factors, we get results of impacted area of dispersion gas from venting process. We use a color mapping to draw the observation area. Different colors determine different values of cell state, which illustrate the concentration of the gas. High concentration of the gas was marked by yellow and low concentration of the gas was marked by blue.

The results of the first case are shown in Figure 3 and Figure 5. We can see from both figures that the gas is moving in consistent direction with the generated wind field while still keep dispersing toward all horizontal direction. The concentration of the gas is also decreasing in the figures as we know that we only observe a specific altitude. Most of the gas is moving upward too, which we cannot see in 2-dimensional images.

We can say that the effect of the wind is quite significant to the movement of gas. It can be seen in Figure 5 and Figure 6 as the result of second case simulation. The gas is dispersing 
slowly and tends to move towards a valley in northeast direction.

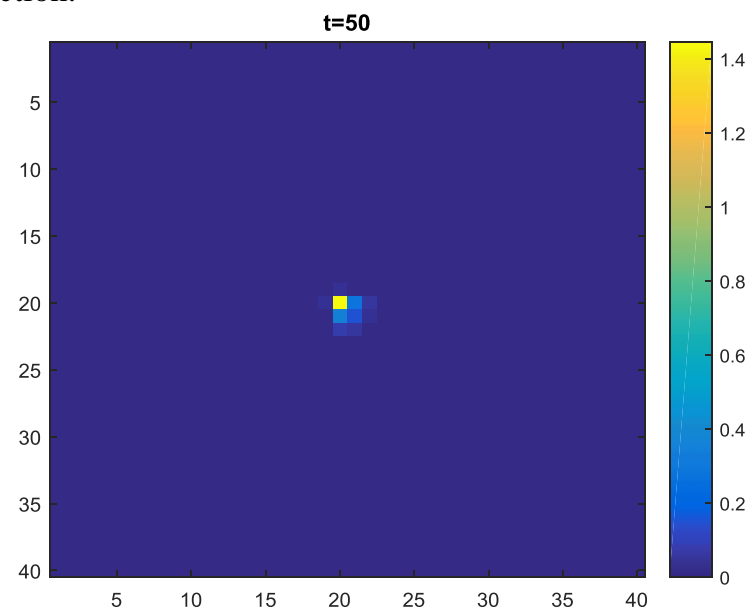

Fig. 3 CA simulation of case 1 based on wind field in the area at time $\mathrm{t}=50$

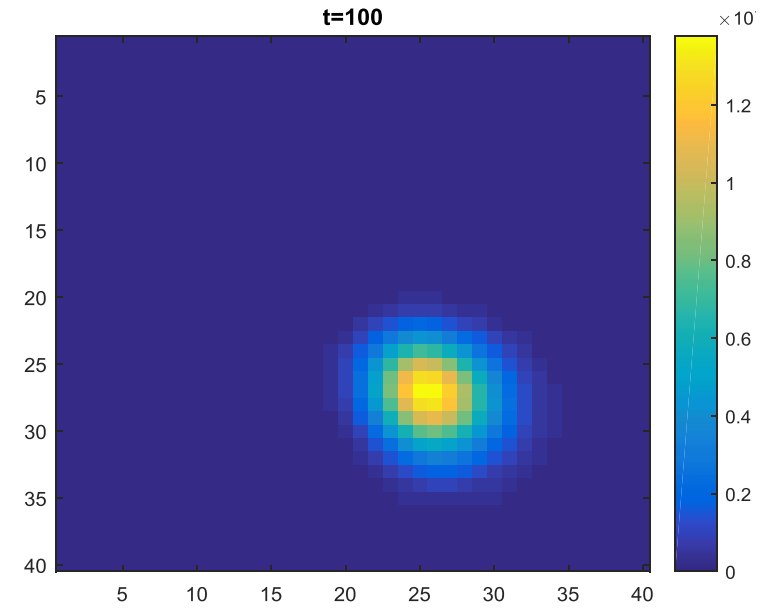

Fig. 4 CA simulation of case 1 based on wind field in the area at time $\mathrm{t}=100$

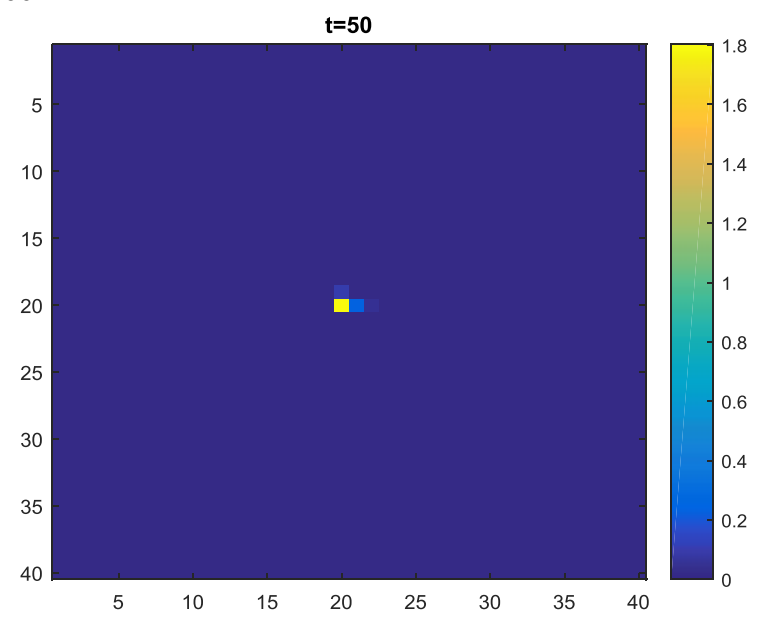

Fig. 5 CA simulation of case 2 based on wind field in the area at time $\mathrm{t}=50$

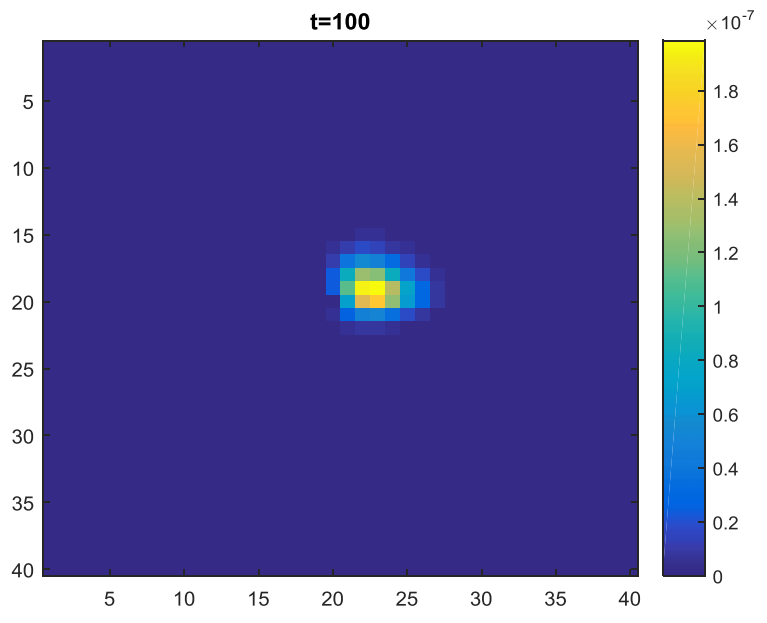

Fig. 7 Eagle view of case 1 based on topography area at time $=100$

\section{V.CONCLUSION}

We have presented a 3-dimensional model of gas concentration dispersion profile released during gas venting process. The 3-dimensional CA is shown to be quite effective in simulating the dispersion towards all directions, without complicated numerical computations. We have shown that the model can be used to predict the impact area of the gas venting process, with information of wind field and land topography around the venting pipe. Some new inputs and development of update rule in this CA model based some more complicated physical factors are possible to improve the accuracy of gas dispersion prediction.

\section{ACKNOWLEDGMENT}

We acknowledge for the support of Research Consortium of OPPINET and all of its institutional members which provide all resources needed for the accomplishment of this study. Our gratitude also goes to Center for Mathematical Modeling and Simulation (PPMS) which has been facilitating RC-OPPINET activities

\section{REFERENCES}

[1] Sarkar, Chinmoy, and Abbasi, S.A, "Cellular automata-based forecasting of the impact of accidental fire and toxic dispersion in process industries", Journal of Hazardous Materials A137, pp.8-30, 2006.

[2] M. Marin, et al, "Cellular Automata Simulation of Dispersion of Pollutants", Computational Materials Science, Vol.18, pp.132-140, 2000 . 